\title{
THE RADIO FREQUENGY SPECTRUM OF CASSIOPEIA A: A SYMPOSIUM SUMMARY
}

Ch. L. SEEGER

University Observatory, Leiden, Netherlands

Data presented at this symposium showed that there had been considerable progress during the previous year towards the solution of one of the basic problems in radio astronomy-the determination of the full radio frequency spectra of the discrete sources. The generally accepted plan is to

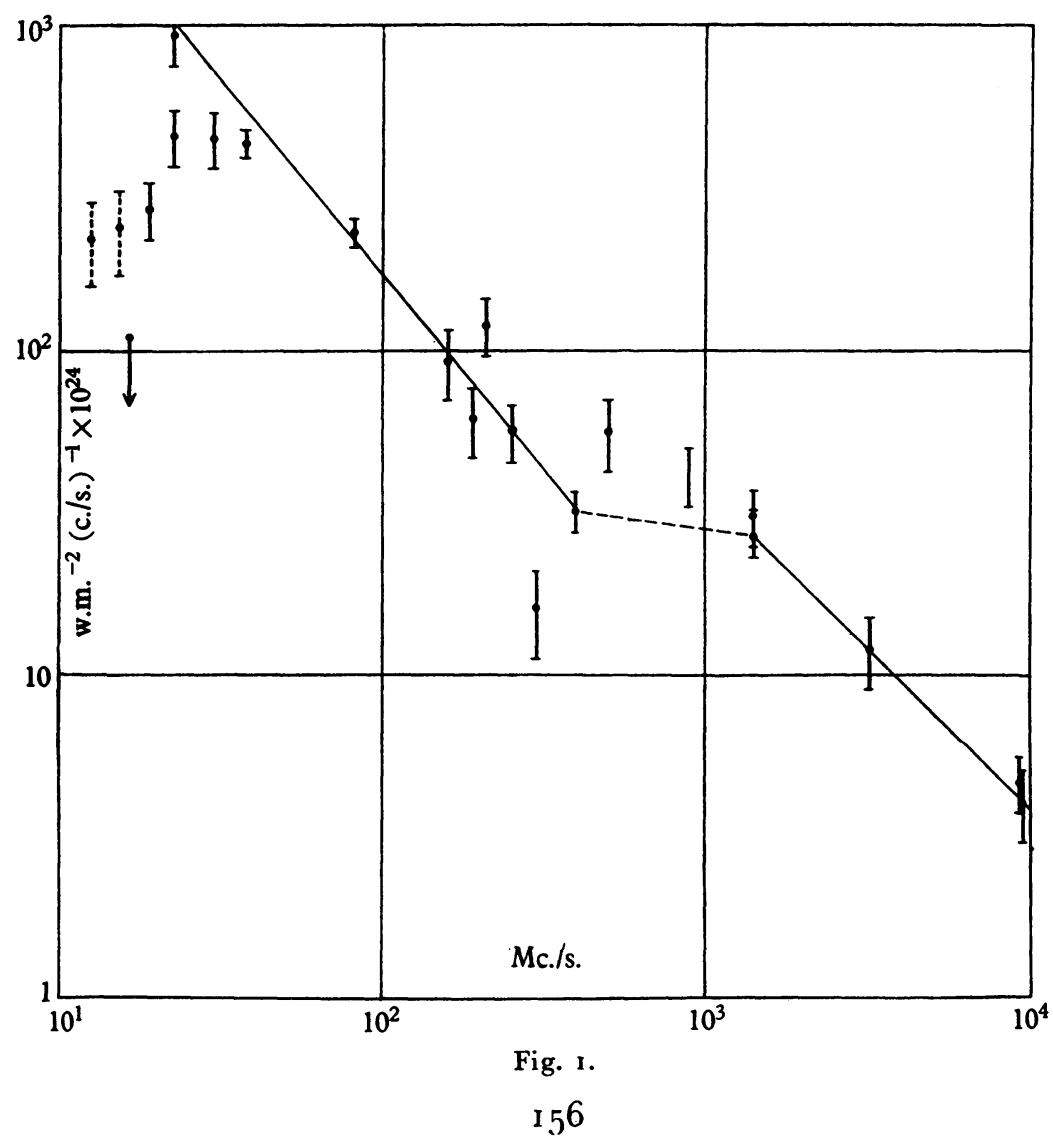


establish the spectra of a few 'reference sources'. At present, these reference objects must be chosen from among the most intense sources, Cassiopeia A, Cygnus A, Taurus A and Virgo A. The utility of both Cygnus A and Taurus A is impaired by their close proximity to other discrete sources of appreciable strength. If they are to be observed with small antennas, meticulous interferometry, employing both variable spacing and variable azimuth, will probably be required in order to obtain an accuracy of $10 \%$. Cassiopeia A and Virgo A appear to be sufficiently in the clear for present needs, though only the latter is visible in both hemispheres and it is about twenty times less bright than Cassiopeia A. However, Cassiopeia A has received most attention so far. The effects of radiometer non-linearity must be examined, particularly at the lower frequencies, when calibrating Virgo A in terms of Cassiopeia A.

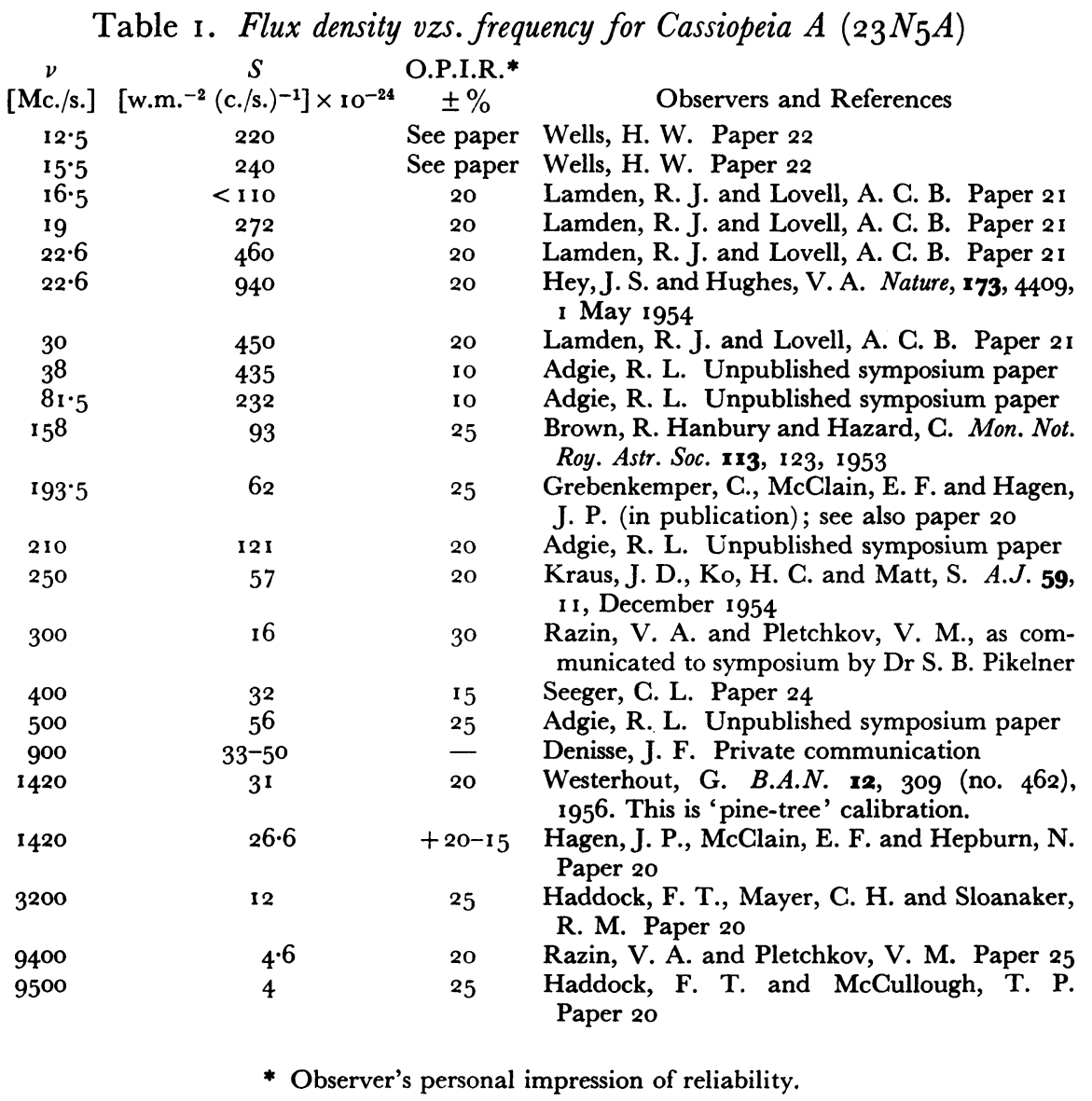


Though the principle of accurate absolute flux density measurement appears to be simple and straightforward, present-day practice has turned out to be unexpectedly difficult. Even with Cassiopeia A, measurements by different observers sometimes disagree by a factor of two or more. Nevertheless, the data on Cassiopeia A seem to be sufficiently consistent, and the spectrum of such intrinsic importance, to warrant the above tabulation and the accompanying curve (Fig. I). I have tried to check each entry with the individual observer, since some of the data are published here for the first time. In the event of any errors or omissions, I herewith tender my most sincere apologies. 